INDUSTRIAL POLICY IN BRITAIN 
Also by David Coates

ECONOMIC AND INDUSTRIAL PERFORMANCE IN EUROPE (editor) THE QUESTION OF UK DECLINE 


\section{Industrial Policy in Britain}

Edited by

\section{David Coates}

Professor of Government and

Co-Director of the International

Centre for Labour Studies

University of Manchester

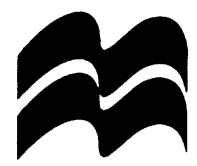


First published in Great Britain 1996 by

MACMILLAN PRESS LTD

Houndmills, Basingstoke, Hampshire RG21 6XS

and London

Companies and representatives

throughout the world

A catalogue record for this book is available

from the British Library.

ISBN 978-0-333-61529-4 ISBN 978-1-349-24570-3 (eBook)

DOI 10.1007/978-1-349-24570-3

First published in the United States of America 1996 by

ST. MARTIN'S PRESS, INC.,

Scholarly and Reference Division,

175 Fifth Avenue,

New York, N.Y. 10010

ISBN 978-0-312-15797-5

Library of Congress Cataloging-in-Publication Data

Industrial policy in Britain / edited by David Coates.

p. $\mathrm{cm}$.

Includes bibliographical references and index.

ISBN 978-0-312-15797-5 (cloth)

1. Industrial policy-Great Britain. I. Coates, David.

HD3616.G73153 1996

$338.941-\mathrm{dc} 20$

95-42142

CIP

(C) Centre for Industrial Policy and Performance, University of Leeds, 1996

All rights reserved. No reproduction, copy or transmission of this publication may be made without written permission.

No paragraph of this publication may be reproduced, copied or transmitted save with written permission or in accordance with the provisions of the Copyright, Designs and Patents Act 1988, or under the terms of any licence permitting limited copying issued by the Copyright Licensing Agency, 90 Tottenham Court Road, London W1P 9HE.

Any person who does any unauthorised act in relation to this publication may be liable to criminal prosecution and civil claims for damages.

$\begin{array}{rrrrrrrrrr}10 & 9 & 8 & 7 & 6 & 5 & 4 & 3 & 2 & 1 \\ 05 & 04 & 03 & 02 & 01 & 00 & 99 & 98 & 97 & 96\end{array}$




\section{Contents}

List of Tables and Figures vii

Notes on the Contributors viii

List of Abbreviations $\quad$ ix

I INTRODUCTION 1

1 Introduction $\quad 3$

David Coates

II SECTORS OF POLICY 31

2 Manufacturing $\quad 33$

Simon Lee

3 Services $\quad 62$

Neill Marshall

4 Energy $\quad 85$

Simon Bromley

5 Agriculture 112

Tom Burden

6 Labour, Skills and Training 135

Alan Tuckman 
7 Whitehall, Westminster and Industrial Policy Kevin Theakston

8 Local and Regional Economic Development

182 Ed Gouge

9 Industrial Policy and the European Union

212 Christopher Lord

\section{CONCLUSIONS}

10 Conclusion

Paul Reynolds and David Coates

Suggested Further Reading

References

271

Index

283 


\section{List of Tables and Figures}

Tables

3.1 Employment and output change, by sector in Britain, 1971-91 63

3.2 Percentage change in service employment in Britain, 1861-1991 64

3.3 The main changes in British financial regulation since $1979 \quad 69$

5.1 EC agriculture and the Common Agricultural Policy $\quad 126$

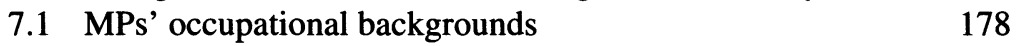

9.1 Proportion of national spending on R\&D by EC Governments

Figures

1.1 Industrial policy and other policy areas 25

1.2 State systems and industrial policy 28 


\section{Notes on the Contributors}

Simon Bromley is Lecturer in Politics, University of Leeds, UK.

Tom Burden is Principal Lecturer, Leeds Business School, Leeds Metropolitan University, UK.

David Coates is Professor of Government at the University of Manchester, UK.

Ed Gouge is Lecturer in Politics, University of Leeds, UK.

Simon Lee is Lecturer in Politics, University of Hull, UK.

Chris Lord is Senior Lecturer in Politics, University of Leeds, UK.

Neill Marshall is Reader in Industrial Geography, Centre for Urban and Regional Development Studies, University of Newcastle, UK.

Paul Reynolds is Senior Lecturer in Contemporary Political Studies, Edge Hill College of Higher Education, Ormskirk, UK.

Kevin Theakston is Senior Lecturer in Politics, University of Leeds, UK.

Alan Tuckman is Senior Lecturer in Sociology, University of Humberside, UK. 


\section{List of Abbreviations}

$\begin{array}{ll}\text { AACP } & \text { Anglo-American Council on Productivity } \\ \text { ACOST } & \text { Advisory Council on Science and Technology } \\ \text { AEI } & \text { Associated Electrical Industries } \\ \text { AGR } & \text { Advanced, Gas-cooled Reactor } \\ \text { BAe } & \text { British Aerospace } \\ \text { BC } & \text { British Coal } \\ \text { BGC } & \text { British Gas Corporation } \\ \text { BL } & \text { British Leyland } \\ \text { BNFL } & \text { British Nuclear Fuels } \\ \text { BNOC } & \text { British National Oil Corporation } \\ \text { BP } & \text { British Petroleum } \\ \text { BT } & \text { British Telecom } \\ \text { CAP } & \text { Common Agricultural Policy } \\ \text { CBI } & \text { Confederation of British Industry } \\ \text { CEGB } & \text { Central Electricity Generating Board } \\ \text { CLA } & \text { Country Landowners Association } \\ \text { CTC } & \text { Central Training Council } \\ \text { DoI } & \text { Department of Industry } \\ \text { DTI } & \text { Department of Trade and Industry } \\ \text { EAGGF } & \text { European Agricultural Guidance and Guarantee Fund } \\ \text { EC } & \text { European Community } \\ \text { EE } & \text { English Electric } \\ \text { EEF } & \text { Engineering Employers Federation } \\ \text { EFTA } & \text { European Free Trade Association } \\ \text { ERDF } & \text { European Regional Development Fund } \\ \text { ESI } & \text { Electricity Supply Industry } \\ \text { ET } & \text { Employment Training } \\ \text { ETSU } & \text { Energy Technology Support Unit } \\ \text { EU } & \text { European Union } \\ & \end{array}$




$\begin{array}{ll}\text { FDI } & \text { Foreign Direct Investment } \\ \text { GATT } & \text { General Agreement on Tariffs and Trade } \\ \text { GDP } & \text { Gross Domestic Product } \\ \text { GEC } & \text { General Electric Company } \\ \text { GNP } & \text { Gross National Product } \\ \text { GPO } & \text { General Post Office } \\ \text { GTC } & \text { Government Training Centre } \\ \text { HMSO } & \text { Her Majesty's Stationery Office } \\ \text { IDC } & \text { Industrial Development Certificate } \\ \text { IDU } & \text { Industrial Development Unit } \\ \text { IRC } & \text { Industrial Reorganisation Corporation } \\ \text { ISDN } & \text { Integrated Services Digital Transmission } \\ \text { IT } & \text { Information Technology } \\ \text { ITB } & \text { Industrial Training Board } \\ \text { LEC } & \text { Local Enterprise Company } \\ \text { MAFF } & \text { Ministry of Agriculture, Fisheries and Food } \\ \text { MITI } & \text { Ministry for International Trade and Industry } \\ \text { MoD } & \text { Ministry of Defence } \\ \text { MSC } & \text { Manpower Services Commission } \\ \text { NAO } & \text { National Audit Office } \\ \text { NATO } & \text { North Atlantic Treaty Organisation } \\ \text { NCB } & \text { National Coal Board } \\ \text { NCVQ } & \text { National Council for Vocational Qualifications } \\ \text { NEB } & \text { National Enterprise Board } \\ \text { NEDC } & \text { National Economic Development Council } \\ \text { NEDO } & \text { National Economic Development Office } \\ \text { NFFO } & \text { Non-fossil Fuel Obligation } \\ \text { NFU } & \text { National Farmers Union } \\ \text { NSTAP } & \text { National Strategic Technology Acquisition Plan } \\ \text { NUM } & \text { National Union of Mineworkers } \\ \text { NVQ } & \text { National Vocational Qualification } \\ \text { OECD } & \text { Organisation for Economic Co-operation and } \\ & \text { Development } \\ \text { OFTEL } & \text { Office of Telecommunications } \\ \text { OPEC } & \text { Organisation of Petroleum Exporting Countries } \\ \text { PWR } & \text { Pressurised Water Reactor } \\ \text { R\&D } & \text { Research and Development } \\ \text { RAF } & \text { Royal Air Force } \\ \text { RDG } & \text { Regional Development Grant } \\ \text { REC } & \text { Regional Electricity Company } \\ & \end{array}$




$\begin{array}{ll}\text { RPI } & \text { Retail Price Index } \\ \text { RSA } & \text { Regional Selective Assistance } \\ \text { SGHWR } & \text { Steam Generating Heavy Water Reactor } \\ \text { SWP } & \text { Sector Working Parties } \\ \text { TEC } & \text { Training and Enterprise Council } \\ \text { TOPS } & \text { Training Opportunities Scheme } \\ \text { TPER } & \text { Total Primary Energy Requirement } \\ \text { UCS } & \text { Upper Clyde Shipbuilders } \\ \text { UKAEA } & \text { United Kingdom Atomic Energy Authority } \\ \text { UKIG } & \text { United Kingdom Industry Group } \\ \text { VANS } & \text { Value-added Network Services } \\ \text { VET } & \text { Vocational and Educational Training } \\ \text { WTO } & \text { World Trade Organisation } \\ \text { YOP } & \text { Youth Opportunities Programme } \\ \text { YT } & \text { Youth Training } \\ \text { YTS } & \text { Youth Training Scheme }\end{array}$

Note: Throughout this book, the term 'European Community' (or EC) refers to anything before the ratification of the Maastricht Treaty in 1993; and 'European Union' (EU) to any subsequent or continuing process, even if begun prior to 1993. 